\title{
Research of Robot Teleoperation System Based on Unity3D Wang $\mathrm{Di}^{1, \mathrm{a}}$, Liu Mouyu ${ }^{2, \mathrm{~b}}$ and Liang Fengyang ${ }^{3, \mathrm{c}}$ \\ ${ }^{1}$ Shanghai Key Laboratory of Intelligent Manufacturing and Robotics Shanghai University Shanghai, China \\ ${ }^{2}$ Shanghai Key Laboratory of Intelligent Manufacturing and Robotics Shanghai University Shanghai, China \\ ${ }^{3}$ Shanghai Key Laboratory of Intelligent Manufacturing and Robotics \\ Shanghai University Shanghai, China \\ adiwang@mail.shu.edu.cn, 'liumouyu@163.com, '2672818993@qq.com
}

Keywords: Unity3D,teleoperation,serial communication, robot

Abstract. Teleoperation technology is a very promising direction in the field of robot.Using virtual reality technology to provide feedback to the operator in teleoperation robot system, which help the operator to know the information of work site and improve the work efficiency.we took the Unity3D as virtual reality simulation platform to achieve the goal of real-time simulation by analyzing the movement of the robot and designing the function of the interface between human and robot.At the same time,the two virtual robot can interact real timely and synchronously by the way of serial communication.

\section{Introduction}

At present,the research of robot teleoperation has become an important research subject.with the development of computer technology and the modern network technology, the technology of graphic simulation and virtual reality provide new thoughts and methods for the research of robotics,especially to improve the remote operation ability of robots that work in the harsh environment.

Virtual reality (VR) is a kind of multi-sensor fusion and multimedia integrated computer systems that can create and experience the virtual world.the operator can control the real robot by manipulating the simulation robot in the virtual environment through the robot teleoperation system that based on virtual reality, which can not only overcome the effect of communication delay,but also provides a friendly human-machine Interface. The monitoring effect is striking by changing the Angle of virtual environment's viewpoint,which can help the operator to complete the task more effectively.

We build the Robot Teleoperation System in the platform of Unity3D,the system can realize the robot's virtual simulation and teleoperation. The method of Communication between virtual robot and real robot is serial communication. Through method of serial communication, the real robot can be controlled by receiving the data from the virtual robot in Unity3D. Visual Studio 2010 is code editor platform and $\mathrm{C \#}$ is the main programming language in this system. We set a 6 DOF joint robot as our research object.The robot's physical diagram is shown in figure 1:

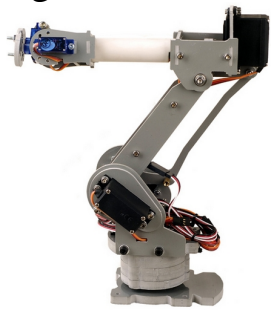

Fig.1The robot's physical diagram 


\section{Establishment of robot's model}

We set Solidworks and 3ds Max as the modeling platform of robot,the specific process is as follows:

At first, the establishment of the robot's geometric model.In order to realize robot's interactive control and enable it to produce movement,the robot's model cannot be established as a whole but should be taken apart in the movement joints and be established as each part respectively according to the robot's DOF.For the robot with six DOF, we divided it into seven parts and established precise modeling for each part.Once the robot's model is established completely in Solidworks, which will be exported by the format of $3 \mathrm{ds}$.

At second,as the model established in Solidworks is just a simple and geometric model.So the model needed to be rendered in 3ds Max for achieving image sense of reality. The model need to be added texture mapping to show more truly and adjusted origin and axis of the parts's movement in 3dsMax.

At third,the model have been rendered will be exported as the format of FBX and then be imported into the Unity3D.Texture will be added to the parts' model in the Unity3D.The modeling process of the robot is shown in figure2.

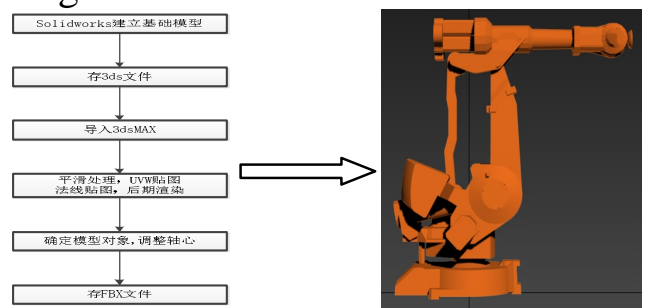

Fig.2 the modeling process of the robot

\section{Simulation and human-computer interaction of robot}

\section{Motion simulation of robot}

The robot is consisted of waist,arm,postbrachium1,2, forearm1,2, connecting rod,wrist and paw. Figure 3 is a diagram for the series-parallel mechanism motion of the robot.The serial mechanism is composed of connecting rod,wrist and paw, which is connected by rotation pair.Postbrachium1,2and forearm1,2constitute a 2DOF parallel mechanism which is used to adjust the beginning position of the robot's posture.

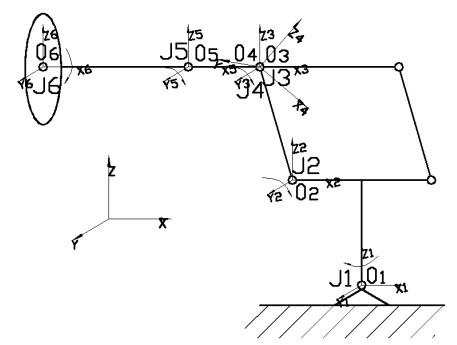

Fig.3diagram for the series-parallel mechanism motion of the robot

The serial mechanism is used to positioning robot's gesture and adjust robot's position.To describe characteristic parameters of each branch and relationship between each branch,we take D-H function to set the coordinate system of each bar.shown in figure3, we set connecting rod coordinate system $O_{4}-X_{4} Y_{4} Z_{4}$ and each branch's coordinate system $O_{5}-X_{5} Y_{5} Z_{5}, O_{6}-X_{6} Y_{6} Z_{6}$ in turn from connecting rod to the end.The linkage parameters of the serial mechanism is shown in table 1.gesture and position of the end of serial mechanism would be changed by changing 12 elements of matrix T3. 
Table 1 the linkage parameters of the serial mechanism

\begin{tabular}{|l|l|l|l|l|}
\hline$i$ & $\theta_{i}$ & $d_{i}$ & $\alpha_{i-1}$ & $a_{i-1}$ \\
\hline 1 & $\theta_{1}$ & 0 & 0 & 0 \\
\hline 2 & $\theta_{2}$ & 0 & 0 & $a_{2}$ \\
\hline 3 & $\theta_{3}$ & 0 & $90^{\circ}$ & $a_{3}$ \\
\hline
\end{tabular}

When the parameters of each joint is determined,T3would be got by homogeneous transformation:

$$
T_{3}=\left[\begin{array}{cccc}
c_{3}\left(c_{1} c_{2}-s_{1} S_{2}\right) & s_{3}\left(s_{1} S_{2}-c_{1} c_{2}\right) & c_{1} S_{2}+s_{1} c_{2} & a_{2}\left(c_{1} c_{2}-s_{1} S_{2}\right)+d_{3}\left(c_{1} S_{2}+s_{1} c_{2}\right)+c_{1} a_{1} \\
c_{3}\left(s_{1} c_{2}+c_{1} S_{2}\right) & -s_{3}\left(s_{1} c_{2}+c_{1} S_{2}\right) & s_{1} S_{2}-c_{1} c_{2} & a_{2}\left(s_{1} c_{2}+c_{1} S_{2}\right)+d_{3}\left(s_{1} S_{2}-c_{1} c_{2}\right)+s_{1} a_{1} \\
s_{3} & c_{3} & 0 & d_{2}+d_{1} \\
0 & 0 & 0 & 1
\end{array}\right]
$$

In type: $c_{i}=\cos \theta_{i}, \quad s_{i}=\sin \theta_{i}$

The parallel mechanism can be set as a four-bar linkage, according to calculation formula of DOF of planar mechanism:

$$
F=3 n-2 P_{L}-P_{H}+P-P_{1}
$$

In type: $F$ - freedom degree, $P_{L}$ —numbers of low pair, $P_{H}$ —numbers of high pair, $n$-numbers of moving link, $P$-numbers of virtual constraint, $P_{1}$-Passive Degree of Freedom.

For the parallel,there are three moving links,four revolute pair and a virtual constraint.so the parallel's DOF is $F=3 \times 3-2 \times 4+1=2$.

\section{Human-computer interaction of robot}

The operator can control the real robot by control the virtual robot through the mouse and keyboard.Interaction is the key to control the robot and the script is the way of realizing interaction.Attaching a script on an empty object can realize the dynamic interaction of 3D scene.

For example, we want to control the rotation of a joint by a key on the keyboard.We set a case that the second connecting part can be controlled to rotate right by the key $\mathrm{W}$, which can easily achieved by the script that is written by $\mathrm{C \#}$ the language:

$$
\begin{gathered}
\text { GameObject a = GameObject.Find("part2"); } \\
\text { float Speed }=3 ; \\
\text { if (Input.GetKey }(\text { KeyCode.W)) } \\
\{ \\
\text { a.transform.Rotate(Vector3.up, Speed, Space.Self); }
\end{gathered}
$$

\begin{tabular}{|c|c|c|}
\hline DEt & 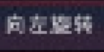 & PAR \\
\hline 问上程 & (A) Fis & PART \\
\hline 问上酉神 & 肉下埴神 & PAR \\
\hline 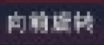 & 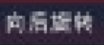 & PAR \\
\hline 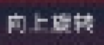 & 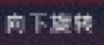 & PAR \\
\hline 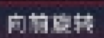 & 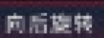 & PA \\
\hline
\end{tabular}

Another important function of interaction is the design of GUI interface.there are a series of button set up in the GUI interface can control the movement of each joint. As shown in figure 4:

Fig.4 GUI interface

The user can control the movement of each joint of the robot by operating the buttons.we also set a case that the second connecting part can be controlled to rotate right by clicking on the button, the corresponding code is as follows.

$$
\begin{gathered}
\text { if (GUI.Button(new Rect(20, 10, 80, 30), "left")) } \\
\text { a.transform.Rotate(Vector3.up, Speed, Space.Self); }
\end{gathered}
$$


The system's overall simulation interface as shown in figure 5.

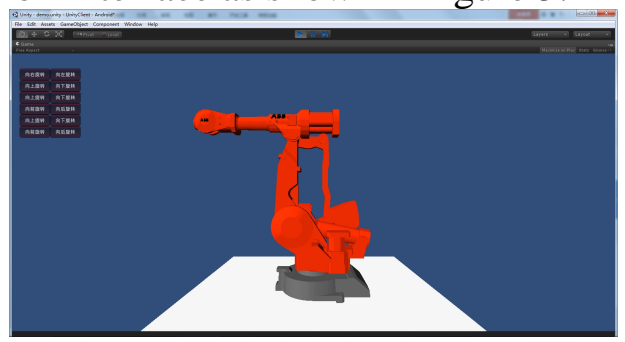

Fig. 5 the effect picture of robot's simulation

\section{The communication of robot teleoperation system based on Unity3D}

The serial communication between Unity and steering gear is realized by the Class SerialPort of Unity.

The first step is to set a serial port and open the port, which is achieved by the function SerialPort () :

public static SerialPort sp = new SerialPort("COM6", 9600, Parity.None, 8, StopBits.One); The second step is to write data, which is achieved by the function serialPort1.WriteLine() : sp.Write(b);

The third step is to add receiving events:

private void serialPort1_DataReceived(object sender, SerialDataReceivedEventArgs e);

Running the platform of Unity3D and lower machine respectively and they can transfer data each other, that means The virtual robot and the real robot can make real-time synchronous movement of the interaction. The effect of real-time synchronous movement of virtual robot and real robot as shown in figure 7.

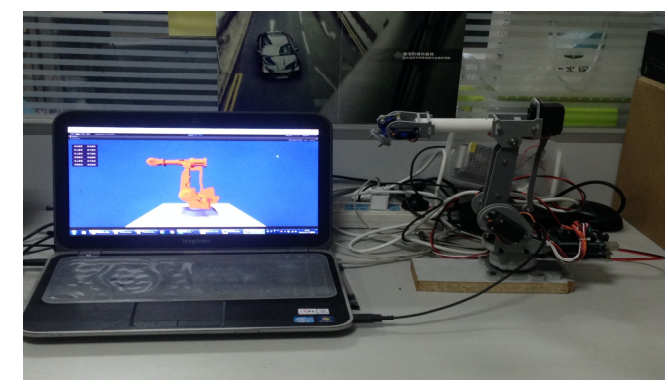

Fig.6 The effect of real-time synchronous movement of virtual robot and real robot

\section{References}

[1] Aswin Indraprastha,MichihIKo Shinozaki. The Investigation on Using Unity3D Game Engine in Urban Design Study[J].ITB Journal of Information and Communication Technology,2009

[2] Graft,Kris.Latest EKI One A.I.Middleware IntegratesWithUnity3D[J].Game Developer,2011

[3] Liu Shouxian,Chen Zongtao, Liu Zhaotong. Virtual reality technology in the application of robot technology and prospects $[\mathrm{J}]$. Journal of information technology and information technology, 2010 (4) : 42-44.

[4] Luo Jie.Simulation of carrying manipulator based on Unity3D [J]. Journal of popular science and technology, 2014 (1) : 62-64.

[5] Zhang Kemin. Robot based on virtual reality simulation study [D]. Chongqing:chongqing 
university, 2012

[6] XULL, YUZR.Communication between SR23 and PC based on serial port in C\#.Net[J].Computer and Modernization,2011(5):107-109

[7] ZHANG J CH.Research and implementation of serial communication strategy based on C\#[J]. Journal of Liao Cheng University,2010,23(1):100-103

[8] Lee Ale, Zheng Xiaowen,Xin Hailin,Zhai Donghan.Hydraulic support movement simulation system based on the Unity $3 \mathrm{~d}$ study $[\mathrm{J}]$ - mechanical and electrical product development and innovation. 2014 (5) : 27

[9] Ry J H,Aritigas J,Preusche C.A passive bilateral control scheme for a teleoperation [J]. Mechatronics.2010,20:812-823 